Journal of Structural Heart Disease, February 2016, Volume 2, Issue 1: 33-34

DOI: http://dx.doi.org/10.12945/j.jshd.2016.012.14
Received: December 11, 2014 Accepted: December 29, 2014 Published online: February 2016

\title{
Current Status of Percutaneous Pulmonic Valve Therapies: The Melody Valve
}

\author{
Marc Gewillig, MD, PhD, FAEPC, FESC, FACC \\ Pediatric Cardiology, Leuven University, UZ Leuven, Herestraat 49, Leuven, Belgium
}

\begin{abstract}
Transcatheter percutaneous pulmonary valve implantation (PPVI) was first described in $\mathbf{2 0 0 0}$ by Philip Bonhoeffer et al 1 as an alternative to open-heart surgery to prolong survival of a right ventricular outflow tract (RVOT) valve conduit.

Since then multiple studies have documented the short term benefits of PPVI implantation using the Melody ${ }^{\mathrm{Tm}}$ valved stent (Medtronic Inc, Minneapolis, USA) for dysfunctional right ventricle to pulmonary artery (RV-AP) conduits.

This review discusses the development, current status and future endeavors of the Melody valve in the pulmonic position.

Copyright $\odot 2016$ Science International Corp.
\end{abstract}

\section{Key Words}

Pulmonic valve therapy • Melody valve

\section{Conflict of Interest}

The author is proctor for Medtronic, Edwards, NuMed.

\section{References}

1. Bonhoeffer P, Boudjemline $Y$, Saliba Z, Merckx J, Aggoun Y, Bonnet D, et al. Percutaneous replacement of pulmonary valve in a right-ventricle to pulmonary-artery prosthetic conduit with valve dysfunction. Lancet 2000;356(9239):1403-1405. DOI: 10.1016/S0140-6736(00)02844-0

2. McElhinney DB, Hellenbrand WE, Zahn
EM, Jones TK, Cheatham JP, Lock JE, et al. Short- and medium-term outcomes after transcatheter pulmonary valve placement in the expanded multicenter US melody valve trial. Circulation 2010;122(5):507516. DOI: 10.1161/CIRCULATIONAHA.109.921692

3. Nordmeyer J, Lurz P, Khambadkone S,
Schievano S, Jones A, McElhinney DB, et al. Pre-stenting with a bare metal stent before percutaneous pulmonary valve implantation: acute and 1-year outcomes. Heart 2011;97(2):118-123. DOI: 10.1136/ hrt.2010.198382

4. McElhinney DB, Cheatham JP, Jones TK, Lock JE, Vincent JA, Zahn EM, et al. Stent
Fax +1 2037853346

E-Mail: jshd@scienceinternational.org

http://structuralheartdisease.org/

\author{
(C) 2016 Journal of Structural Heart Disease \\ Published by Science International Corp. \\ ISSN 2326-4004 \\ Accessible online at: \\ http://structuralheartdisease.org/
}

* Corresponding Author:

Marc Gewillig, MD, PhD, FAEPC, FESC, FACC Pediatric Cardiology Leuven University

UZ Leuven, Herestraat 49, Leuven, Belgium

Tel.: +32 16 343865; Fax: +32 16 343981; E-Mail: marc.gewillig@uzleuven.be 
fracture, valve dysfunction, and right ventricular outflow tract reintervention after transcatheter pulmonary valve implantation: patient-related and procedural risk factors in the US Melody Valve Trial. Circulation. Cardiovascular interventions 2011;4(6):602-614. DOI: 10.1161/CIRCINTERVENTIONS.111.965616

5. Feltes TF, Bacha E, Beekman RH 3rd, Cheatham JP, Feinstein JA, Gomes AS, et al. American Heart Association Congenital Cardiac Defects Committee of the Council on Cardiovascular Disease in the Young; Council on Clinical Cardiology; Council on Cardiovascular Radiology and Intervention; American Heart Association. Indications for cardiac catheterization and intervention in pediatric cardiac disease: a scientific statement from the American Heart Association. Circulation. 2011;7;123(22):2607-2652. DOI: 10.1161/ CIR.0b013e31821b1f10

6. Boshoff DE, Cools BL, Heying R, Troost E, Kefer J, Budts W, et al. Off-label use of percutaneous pulmonary valved stents in the right ventricular outflow tract: time to rewrite the label? Catheterization and cardiovascular interventions : official journal of the Society for Cardiac Angiography
\& Interventions 2013;81(6):987-995. DOI: $10.1002 / \mathrm{ccd} .24594$

7. Gillespie MJ, Rome JJ, Levi DS, Williams RJ, Rhodes JF, Cheatham JP, et al. Melody valve implant within failed bioprosthetic valves in the pulmonary position: a multicenter experience. Circ Cardiovasc Interv. 2012;5(6):862-870. DOI: 10.1161/ CIRCINTERVENTIONS.112.972216

8. Meadows JJ, Moore PM, Berman DP, Cheatham JP, Cheatham SL, Porras D, et al. Use and performance of the Melody Transcatheter Pulmonary Valve in native and postsurgical, nonconduit right ventricular outflow tracts. Circ Cardiovasc Interv. 2014;7(3):374-380. DOI: 10.1161/ CIRCINTERVENTIONS.114.001225

9. Morray BH, McElhinney DB, Cheatham JP, Zahn EM, Berman DP, Sullivan PM, et al. Risk of coronary artery compression among patients referred for transcatheter pulmonary valve implantation: a multicenter experience. Circulation. Cardiovascular interventions 2013;6(5):535542. DOI: $10.1161 /$ CIRCINTERVENTIONS.113.000202

10. Nordmeyer J, Khambadkone S, Coats L, Schievano S, Lurz P, Parenzan G, et al. Risk stratification, systematic classification, and anticipatory management strategies for stent fracture after percutaneous pulmonary valve implantation. Circulation 2007;115(11):1392-1397. DOI: 10.1161/ CIRCULATIONAHA.106.674259

11. Cools B, Budts W, Heying R, Boshoff D, Eyskens B, Frerich S, Troost E, Gewillig M. Medium term follow-up after percutaneous pulmonary valve replacement with the Melody ${ }^{\circledR}$ valve. IJC Heart \& Vasculature 2015;7:92-97. DOI: 10.1016/j. ijcha.2015.02.014

12. Van Dijck I, Budts W, Cools B, Eyskens B, Boshoff DE, Heying $R$, et al. Infective endocarditis of the Melody valved stent in comparison to surgical implants in Right Ventricular Outflow Tract. Heart. 2015;101(10):788-93. DOI: 10.1136/heartjnl-2014-306761

Cite this article as: Gewillig M. Current Status of Percutaneous Pulmonic Valve Therapies: The Melody Valve. Journal of Structural Heart Disease 2015;1(2):3334. DOI: http://dx.doi.org/10.12945/j. jshd.2015.012.14 